\title{
Efficiency and equity analysis of toll pricing on Sydney Harbour Bridge with heterogeneous travellers
}

\author{
S. Wang ${ }^{a *}$ \\ M. Dunbar ${ }^{b^{*}}$ \\ M. Harrison ${ }^{b}$
}

\begin{abstract}
Sydney Harbour Bridge is a key transport infrastructure that connects North Sydney and Sydney Central Business District (CBD). To alleviate the congestion on Sydney Harbour Bridge, NSW Roads and Maritime Services imposes a time of day tolling between $\$ 2.5$ and $\$ 4$ on the southbound traffic to Sydney CBD. This study develops mathematical models for formulating the toll pricing problem on Sydney Harbour Bridge considering that different travellers may have different value-of-times (VOTs). The models examine quantitatively the effect of different toll levels on the efficiency (in terms of the total generalized travel time and generalized travel cost of all travellers) and equity (in terms of the ratio of generalized travel cost among different traveller classes). The proposed models can serve as a useful decision-support tool for NSW Roads and Maritime Services.
\end{abstract}

Key words: Toll pricing; Traffic equilibrium; Heterogeneous value-of-time; Sydney Harbour Bridge; Optimization.

\section{Introduction}

In view of the importance of the Sydney Harbour Bridge on transport efficiency in Sydney, this paper develops mathematical models for formulating the toll pricing problem on Sydney Harbour Bridge considering that different travellers may have different value-of-times (VOTs). The models examine quantitatively the effect of different toll levels on the efficiency and equity. The objective is to provide a useful decision-support tool for NSW Roads and Maritime Services.

\section{Toll pricing with homogeneous travellers}

We assume that there are two roads from North Sydney to CBD of Sydney: one via Sydney Harbour Bridge (link $a$ ) and the other via other bridges (link $b$ ), see Figure 1. Link $b$ is longer and has a larger capacity than link $a$. The link travel time functions are assumed to be of the Bureau of Public Roads (BPR) form:

\footnotetext{
${ }^{a}$ Lecturer, Ph.D. School of Mathematics and Applied Statistics, University of Wollongong, Wollongong, NSW 2522, Australia. Email: wangshuaian@gmail.com

${ }^{\mathrm{b}}$ SMART Infrastructure Facility, University of Wollongong, Wollongong, NSW 2522, Australia,

*Corresponding Author: Michelle Dunbar mdunbar@uow.edu.au

http://dx.doi.org/10.14453/isngi2013.proc.47
} 


$$
t_{i}\left(v_{i}\right)=t_{i}^{0}\left[1+\alpha_{i}\left(\frac{v_{i}}{C_{i}}\right)^{\beta_{i}}\right], i \in\{a, b\}
$$

where $t_{i}^{0}$ is the free-flow travel time (minutes), $v_{i}(\mathrm{vph})$ is the traffic flow, $C_{i}$ (vph) is the link flow capacity, and $\alpha_{i}>0$ and $\beta_{i}>1$ are two parameters.

We represent by $q$ (vph) the travel demand from North Sydney to Sydney CBD, and we denote by $v_{a}$ and $v_{b}$ the flow on link $a$ and $b$, respectively. The link flow must satisfy flow conservation equations. For simplicity, we define a set:

$$
\Omega=\left\{\left(v_{a}, v_{b}\right) \mid v_{a}+v_{b}=q, v_{a} \geq 0, v_{b} \geq 0\right\}
$$

containing all the possible link flow vector $v:=\left(v_{a}, v_{b}\right) \in \Omega$.

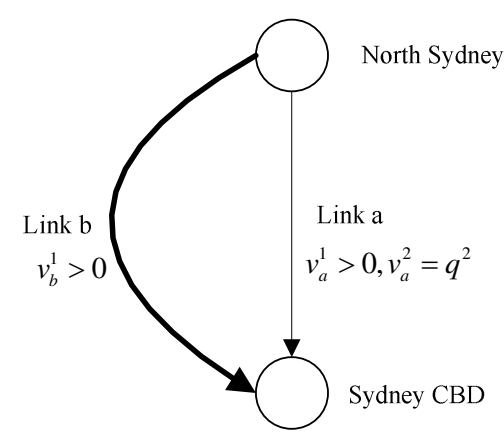

(i) $\tau_{a}<0.63$

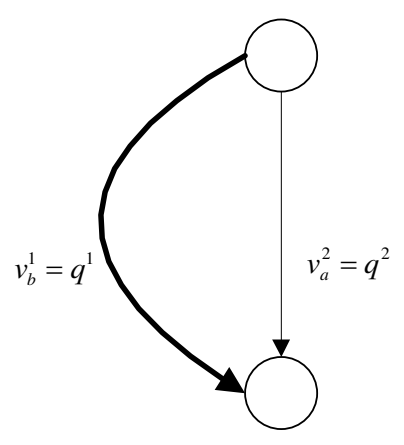

(ii) $0.63 \leq \tau_{a} \leq 1.88$

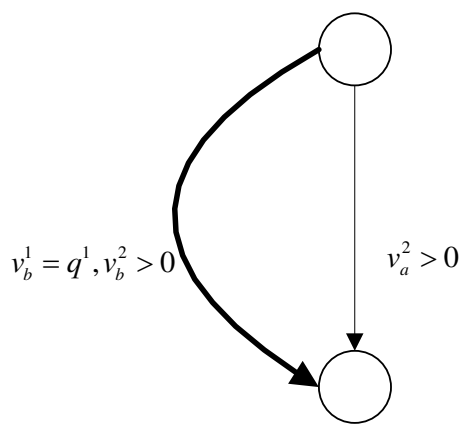

(iii) $\tau_{a}>1.88$

Figure 1. Transport flow with different tolls.

\section{A. User equilibrium, system optimum, and optimal toll pricing}

Since each traveller aims to minimize her/his travel time, at the user equilibrium (UE), if no toll is imposed, the travel times on the two links are identical $t_{a}\left(v_{a}\right)=t_{b}\left(v_{b}\right)$. We assume that all travelers are homogeneous with value of time (VOT) $\gamma(\$ / \mathrm{min})$. If the government imposes toll on both links denoted by $\tau:=\left(\tau_{a}, \tau_{b}\right)$, the equilibrium is:

$$
\gamma t_{a}\left(v_{a}\right)+\tau_{a}=\gamma t_{b}\left(v_{b}\right)+\tau_{b}
$$

We can compute the resulting link flow $v_{a}$ and $v_{b}$ denoted by $v^{U E}$.

At system optimum (SO), the total travel time of all road users is minimized, and the SO link flow is denoted by $v^{S O}$. The optimal SO toll denoted by $\tau^{*}$ can be computed by the marginal social cost theory. Alternatively, the government may impose a non-zero toll on only one link by requiring that: 


$$
\begin{aligned}
& \tau_{a}=\tau_{a}^{*}-\min \left\{\tau_{a}^{*}, \tau_{b}^{*}\right\} \\
& \tau_{b}=\tau_{b}^{*}-\min \left\{\tau_{a}^{*}, \tau_{b}^{*}\right\}
\end{aligned}
$$

\section{B. An example using Sydney Harbour Bridge}

We use the case of Sydney Harbour Bridge to demonstrate the above models. The free-flow travel time of Sydney Harbour Bridge is calculated as follows. It is $1.149 \mathrm{~km}$ long and we assume a free-flow travel speed of $80 \mathrm{~km} / \mathrm{h}$. Hence, $t_{a}^{0}=0.86 \mathrm{~min}$. It has 8 traffic lanes controlled by Electronic Lane Changing System (ELCS). Therefore we assume that 6 lanes are used in the southbound direction during morning peak. We further assume that the capacity of one lane is 2000 vehicles/h. Consequently, $C_{a}=12,000$. The coefficients $\alpha_{a}=0.15$ and $\beta_{a}=$ 2. We assume that the length of link $b$ is $5 \mathrm{~km}$, its free-flow travel speed is $80 \mathrm{~km} / \mathrm{h}$, and it has 20 lanes. Therefore, $t_{b}^{0}=3.75$ and $C_{b}=40,000$. The coefficients $\alpha_{b}=0.15$ and $\beta_{a}=4$. We assume $\beta_{b}>\beta_{a}$ because the traffic on Sydney Harbour Bridge is well controlled and regulated. We assume that the demand in the peak hour is $120,000 \mathrm{vph}$. The value of time $\gamma=0.5 \$ / \mathrm{min}$. The results are reported in Table 1, where the row "Toll ( $\$ 4)$ " means a toll of $\$ 4$ is charged on Sydney Harbour Bridge and the column "TTT" means the total travel time of all travelers as defined below:

$$
T T T=v_{a} t_{a}\left(v_{a}\right)+v_{b} t_{b}\left(v_{b}\right)
$$

When $\$ 4$ is charged (which is the actual toll charge during morning and evening peak hours on weekdays), the flow on Sydney Harbour Bridge is 45,050. To achieve the SO traffic flow, the optimal toll charge on Sydney Harbour Bridge should be $\$ 0.36$. The toll charge $\$ 4$ is apparently too high, and the resulting TTT is not only larger than that under SO, but also significantly larger than that without any intervention (UE).

Table 1. Results with homogeneous travellers.

\begin{tabular}{crrrrrr}
\hline & $v_{a}$ & $v_{b}$ & $t_{a}\left(v_{a}\right)$ & $t_{b}\left(v_{b}\right)$ & $\tau_{a}$ & TTT \\
\hline No toll $(\mathrm{UE})$ & 69,422 & 50,578 & 5.188 & 5.188 & 0 & 622,550 \\
SO & 66,480 & 53,520 & 4.829 & 5.553 & $0.36^{\mathrm{c}}$ & 618,219 \\
Toll $(\$ 4)$ & 45,050 & 74,950 & 2.684 & 10.684 & 4 & 921,642 \\
\hline
\end{tabular}

${ }^{\mathrm{c}}$ This is the optimal $\tau_{a}$ to achieve SO with $\tau_{b}=0$. 


\section{Toll pricing with heterogeneous travelers}

We examine heterogeneous travellers in this section. We consider $M$ traveller classes, and the VOT of class $m=1,2, \ldots, M$ is denoted by $\gamma^{m}(\$ / \mathrm{min})$. The demand of class $m$ is $q^{m}$. The number of travellers from class $m$ on link $i$ is denoted by $v_{i}^{m}$. For simplicity, we define a set:

$$
\hat{\Omega}=\left\{\left(v_{a}^{m}, v_{b}^{m}, m=1,2 \cdots M\right) \mid v_{a}^{m}+v_{b}^{m}=q^{m}, v_{a}^{m} \geq 0, v_{b}^{m} \geq 0, \forall m\right\}
$$

containing all the possible link flow vector $v:=\left(v_{a}^{m}, v_{b}^{m}, m=1, \ldots, M\right) \in \widehat{\Omega}$.

\section{User equilibrium, system optimum, and optimal toll pricing}

With heterogeneous travellers, there are two types of SO: SO in cost units and SO in time units. The SO model in cost units can be formulated as:

$$
\min \sum_{m=1}^{M} \gamma^{m}\left[v_{a}^{m} t_{a}\left(v_{a}\right)+v_{b}^{m} t_{b}\left(v_{b}\right)\right]
$$

According to Ref. 1, the optimal toll can be computed.

\section{An example using Sydney Harbour Bridge}

We look at the Sydney Harbour Bridge example again, and assume two classes: $\gamma^{1}=0.25$, $\gamma^{2}=0.75, q^{1}=60,000$ and $q^{2}=60,000$. Therefore the total demand and the average VOT are the same as sub-section II.B.

The results are reported in Table 2, where the column "Toll (\$4)" means a toll of $\$ 4$ is charged on Sydney Harbour Bridge. The column "TTT" means the total travel time of all travellers defined by Eq. (6). The column "TTC" means the total travel cost of all travellers defined below:

$$
T T C=\sum_{m=1}^{M} \gamma^{m}\left[v_{a}^{m} t_{a}\left(v_{a}\right)+v_{b}^{m} t_{b}\left(v_{b}\right)\right]
$$

We can see that to minimize total travel cost, a toll of $\$ 1.92$ should be imposed on Sydney Harbour Bridge, and only travellers in class 2 use Sydney Harbour Bridge. Both travellers in class 1 and class 2 use other bridges, and hence their generalized travel time is the same, and their generalized travel cost is proportional to their VOTs. If we minimize the total travel time, then slightly fewer people use Sydney Harbour Bridge than the case of no toll (UE). To this end, a toll of $\$ 0.18$ is imposed and therefore all travellers in class 2 use Sydney Harbour Bridge. Travellers in class 1 use both links. Since travellers in class 1 and class 2 have the same travel time and pay the same toll on Sydney Harbour Bridge, the ratio of the generalized travel cost between class 2 and class 1 is reduced from 3 to 2.740 .

When a toll of $\$ 4$ is imposed on Sydney Harbour Bridge, then only travellers in class 2 use it. This case is similar to the case of Cost SO and since both travellers in class 1 and class 2 use the untolled link, their generalized travel time is identical. 
Table 2. Results with heterogeneous travellers.

\begin{tabular}{ccccc}
\hline & No toll (UE) & Cost SO & Time SO & Toll (\$4) \\
\hline$v_{a}^{1}$ & 34,711 & 0 & 6,480 & 0 \\
$v_{a}^{2}$ & 34,711 & 59,819 & 60,000 & 51,531 \\
$v_{b}^{1}$ & 25,289 & 60,000 & 53,520 & 60,000 \\
$v_{b}^{2}$ & 25,289 & 181 & 0 & 8,469 \\
$t_{a}\left(v_{a}\right)$ & 5.188 & 4.074 & 4.829 & 3.245 \\
$t_{b}\left(v_{b}\right)$ & 5.188 & 6.632 & 5.553 & 8.579 \\
$\tau_{a}$ & 0 & $1.92^{\mathrm{d}}$ & $0.18^{\mathrm{e}}$ & 4 \\
Generalized time for class 1 & 5.188 & 6.632 & 5.553 & 8.579 \\
Generalized time for class 2 & 5.188 & 6.632 & 5.070 & 8.579 \\
Generalized cost for class 1 & 1.297 & 1.658 & 1.388 & 2.145 \\
Generalized cost for class 2 & 3.891 & 4.974 & 3.803 & 6.434 \\
Ratio of cost of class 2 over cost of class 1 & 3.000 & 3.000 & 2.740 & 3.000 \\
TTT & 662,550 & 642,824 & 618,219 & 754,636 \\
TTC & 311,276 & 283,153 & 308,421 & 308,607 \\
\hline
\end{tabular}

\section{E. Impact analysis of different toll levels}

We further analyse the flow pattern under different toll levels, and the results are shown in Figure 1. When $\tau_{a}<0.63$, travelers in class 1 and all travellers in class 2 use Sydney Harbour Bridge and experience the same travel time and toll; some travellers in class 1 use other bridges. Therefore, the ratio of the generalized travel cost of class 2 and class 1 is between 3 and 2.24 (less than the ratio of their VOTs). When $0.63 \leq \tau_{a} \leq 1.88$, all travellers in class 2 use Sydney Harbour Bridge and all travellers in class 1 use other bridges. The ratio of the generalized travel cost is between 2.24 and 3.00. When $\tau_{a}>1.88$, some travellers in class 2 use Sydney Harbour Bridge; all travellers in class 1 and the other travellers in class 2 use other bridges. Therefore, on link $b$, the two classes of travellers experience the same travel time and zero tolls. The ratio of the generalized travel cost is 3.00 .

\section{Conclusions}

This paper has developed mathematical models for formulating the toll pricing problem on Sydney Harbour Bridge considering that different travellers may have different value-of-times. The models examine quantitatively the effect of different toll levels on the efficiency and equity.

\footnotetext{
${ }^{\mathrm{d}}$ This is the optimal $\tau_{a}$ to achieve Cost SO with $\tau_{b}=0$.

${ }^{\mathrm{e}}$ This is the optimal $\tau_{a}$ to achieve Cost SO with $\tau_{b}=0$.
} 
International Symposium for Next Generation Infrastructure October 1-4, 2013, Wollongong, Australia

The proposed models can serve as a useful decision-support tool for NSW Roads and Maritime Services.

\section{References}

${ }^{1}$ Yang, H., Huang, H.-J., "The multi-class, multi-criteria traffic network equilibrium and systems optimum problem", Transportation Research Part B, Vol. 38, No. 1, 2004, pp. 1-15. http://dx.doi.org/10.1016/S0191-2615(02)00074-7 